\title{
Biofabricated Structures Reconstruct Functional Urinary Bladders in Radiation-injured Rat Bladders
}

\author{
Tetsuya Imamura $\mathrm{PhD}^{1}$, Mitsuru Shimamura $\mathrm{PhD}^{2}$, Teruyuki Ogawa $\mathrm{MD}, \mathrm{PhD}^{1}$, \\ Tomonori Minagawa MD, $\mathrm{PhD}^{1}$, Takashi Nagai MD, $\mathrm{PhD}^{1}$, Gautam Silwal Sudha ${ }^{1}$, \\ and Osamu Ishizuka MD, $\mathrm{PhD}^{1}$
}

1. Department of Urology, Shinshu University School of Medicine, Nagano, JAPAN

2. Cyfuse Biomedical K.K., Tokyo, JAPAN

Running Head: Biofabricated Bone Marrow-derived Cell Structure 
Corresponding author: Tetsuya Imamura, PhD, Department of Urology, Shinshu University School of Medicine, 3-1-1 Asahi, Matsumoto, Nagano, 390-8621, JAPAN; telephone: +81-263-37-2661; fax: +81-263-37-3082; e-mail: imatetu@shinshu-u.ac.jp

Mitsuru Shimamura PhD, Cyfuse Biomedical K.K., University of Tokyo Entrepreneur Plaza, 7-3-1 Hongo, Bunkyou-ku, Tokyo, 113-0033, JAPAN; telephone: +81-3-4455-7872; e-mail: mitsuru.shimamura@cyfusebm.com

Teruyuki Ogawa MD, PhD, Department of Urology, Shinshu University School of Medicine, 3-1-1 Asahi, Matsumoto, Nagano, 390-8621, JAPAN; telephone: +81-263-37-2661; fax: +81-263-37-3082; e-mail: ogawat@shinshu-u.ac.jp

Tomonori Minagawa MD, PhD, Department of Urology, Shinshu University School of Medicine, 3-1-1 Asahi, Matsumoto, Nagano, 390-8621, JAPAN; telephone: +81-263-37-2661; fax: +81-263-37-3082; e-mail: minagawat@shinshu-u.ac.jp

Takashi Nagai MD, PhD, Department of Urology, Shinshu University School of Medicine, 3-1-1 Asahi, Matsumoto, Nagano, 390-8621, JAPAN; telephone: +81-263-37-2661; fax: +81-263-37-3082; e-mail: ttnagai@shinshu-u.ac.jp

Gautam Silwal Sudha, Department of Urology, Shinshu University School of Medicine, 3-1-1 Asahi, Matsumoto, Nagano, 390-8621, JAPAN; telephone: +81-263-37-2661; fax: +81-263-37-3082; e-mail: kanchu7@gmail.com

Osamu Ishizuka MD, PhD, Department of Urology, Shinshu University School of Medicine, 3-1-1 Asahi, Matsumoto, Nagano, 390-8621, JAPAN; telephone: +81-263-37-2661; fax: +81-263-37-3082; e-mail: ishizuk@shinshu-u.ac.jp 


\section{Abstract}

The ability to repair damaged urinary bladders through the application of bone marrow-derived cells is in the earliest stages of development. We investigated the application of bone marrow-derived cells to repair radiation-injured bladders. We used a three-dimensional (3D) bioprinting robot system to biofabricate bone marrow-derived cell structures. We then determined if the biofabricated structures could restore the tissues and functions of radiation-injured bladders. The bladders of female 10-week-old Sprague-Dawley (SD) rats were irradiated with 2-Gy once a week for 5 weeks. Adherent and proliferating bone marrow-derived cells harvested from the femurs of male 17-week-old green fluorescence protein-transfected Tg-SD rats were cultured in collagen-coated flasks. Bone marrow-derived cell spheroids were formed in 96-well plates. Three layers of spheroids were assembled by the bioprinter onto a 9x9 microneedle array. The assembled spheroids were perfusion cultured for 7 days, and then the microneedle array was removed. Two weeks after the last radiation treatment, the biofabricated structures were transplanted into an incision on the anterior wall of the bladders $(n=10)$. Control rats received the same surgery but without the biofabricated structures (sham-structure, $n=12$ ). At 2 and 4 weeks after surgery, the sham-structure control bladder tissues exhibited disorganized smooth muscle layers, decreased nerve cells, and significant fibrosis with increased presence of fibrosis-marker P4HB-positive cells and hypoxia-marker HIF1 $\alpha$-positive cells. The transplanted structures survived within the recipient tissues, and blood vessels extended within them from the recipient tissues. The bone marrow-derived cells in the structures differentiated into smooth muscle cells and formed smooth muscle clusters. The recipient tissues near the transplanted structures had distinct smooth muscle layers and reconstructed nerve cells, and only minimal 
fibrosis with decreased presence of P4HB- and HIF1 $\alpha$-positive cells. At 4 weeks after surgery, the sham-structure control rats exhibited significant urinary frequency symptoms with irregular and short voiding intervals, and low micturition volumes. In contrast, the structure-transplanted rats had regular micturition with longer voiding intervals and higher micturition volumes compared to the control rats. Further, the residual volume of the structure-transplanted rats was lower than for the controls. Therefore, transplantation of biofabricated bone marrow-derived cell structures reconstructed functional bladders. 


\section{Introduction}

Previously, we showed that adherent proliferating bone marrow-derived cells harvested from femurs have the potential to reconstruct functional urinary bladders from damaged ones $(1,2)$. To deliver the cells into recipient bladders, we utilized two methods. One was the direct-injection of single cells (1) and the other was patch-transplantation of cell sheets produced in temperature-responsive culture dishes (2). Both methods successfully reconstructed bladder tissues and restored much of the functionality. However, there are some limitations for each method. In the direct-injection method, survival rates of the implanted cells were very low within the recipient tissues. For the cell sheet patch-transplantation method, the sheets were too thin and weak to be reliably delivered to the recipient tissues. To overcome these limitations, we have attempted to biofabricate bone marrow-derived cell structures with a three-dimensional (3D) bioprinting robot system.

Novel 3D bioprinter robotic systems can now provide biofabrication processes to construct tissue-like structures $(3,4)$. The bioprinters apply individual drops of "bioink" that consist of prepolymer solutions, cells/cell aggregates (spheroids), or both mixed suspensions. Three different types of printers currently exist: inkjet (5-10), microextrusion (11-14), and laser-assisted bioprinting (15-22). Most of these bioprinting techniques need to be supported with (bio-)material scaffolds to biofabricate tissue-like structures. In this study, we used a 3D bioprinting robot system that picks up and assembles spheroids according to the desired 3D design. Thus, this 3D bioprinting robot system was classified as inkjet and/or microextrusion bioprinter. However, the biofabricated structures were finally formed without any supporting (bio-)material scaffolds, and thus they consisted of only the assembled, self-organized 
spheroids. Importantly, the structures had sufficient thickness and strength to be directly transplanted into the recipient bladder tissues.

In urology, low compliance bladder and/or contracted bladder, either of which can occur as a result of various diseases such as spina bifida, spinal column injury, diabetes, or interstitial cystitis, are considered to be refractory lower urinary tract symptoms (LUTS). Treatments for the terminal phase of low compliance and/or contracted bladder are bladder expansion or urinary diversion through the intestinal tract. However, these operations are highly invasiveness, and urinary diversion has several side effects resulting from utilization of the intestinal tract. Therefore, novel treatments for low compliance and/or contracted bladder are urgently needed.

In this study, we used radiation-injured rat urinary bladders to create the conditions of low compliance and/or contracted bladders. Previously, we established that the radiation injured bladders have a similar histological appearance and manifest similar bladder dysfunctions as these conditions (1,2). We first documented the histological and immunohistochemical findings of the bone marrow-derived cells in the biofabricated structures within the radiation-injured rat bladders. We then determined if the transplanted biofabricated structures could restore the structural organization and functionality of the damaged bladders. 


\section{Material and Methods}

Animals

Twenty-two female 10-week-old Sprague-Dawley (SD) rats (Japan SLC Inc., Shizuoka, Japan) were used as recipients. Six male 17-week-old green fluorescence protein (GFP)-transfected Tg-SD rats (Japan SLC Inc.) were used as bone marrow cell donors. All of the rats were maintained with freely available food and water under a 12-hour alternating light-dark cycle. After each experiment, the rats were euthanized by an overdose of pentobarbital sodium solution (Kyoritsu Seiyaku Co., Tokyo, Japan). All animals were treated in accordance with National Institutes of Animal Health Care Guidelines and the guidelines approved by the Animal Ethics Committee of Shinshu University School of Medicine.

\section{Production of Radiation-injured Urinary Bladders}

Radiation injury to the urinary bladders was produced as we previously described $(1,2)$. Briefly, the recipient SD rats were anesthetized with $40 \mathrm{mg} / \mathrm{kg}$-body weight pentobarbital sodium solution (Kyoritsu Seiyaku Co.), and then protected with an iron shield except for a 1-cm diameter circle bordering on the pubic bone to allow radiation of the pelvic region containing the bladder. The exposed area was irradiated with 2-Gy once a week for 5 weeks. Following the last radiation exposure, the rats were maintained for 2 weeks. Three days prior to transplantation, they received immunosuppression treatments with $15 \mathrm{mg} / \mathrm{kg}$-body weight cyclosporin (Novartis International AG, Basle, Switzerland) and $2 \mathrm{mg} / \mathrm{kg}$-body weight 6 $\alpha$-methylprednisolone (Sigma-Aldrich, St. Louis, MO, USA) by subcutaneous injection every day. Two weeks after the last radiation treatment, they were used as recipient animals. 
Biofabrication of Bone Marrow-derived Cell Structures

The bone marrow-derived cells were prepared according to our previous report $(1,2)$. Briefly, both femurs of the donor GFP-transfected Tg-SD rats were harvested and flushed with $10 \mathrm{ml}$ of culture medium composed of Dulbecco's Modified Eagle Medium (DMEM) high-glucose (Gibco, Thermo Fisher Scientific K.K., Kanagawa, Japan) supplemented with 15\% regular fetal bovine serum (Biowest, Nuaille, France) and $0.1 \%$ penicillin-streptomycin solution (Gibco). The cells were seeded and cultured onto type I collagen-coated 10-cm culture dishes (Asahi Techno Glass, Shizuoka, Japan) for 7 days. When the cells achieved confluency, the adherent and proliferating bone marrow-derived cells were transferred into type I collagen-coated 225- $\mathrm{cm}^{2}$ culture flasks (Asahi Techno Glass) for subculture. To obtain enough cells, establishment of third and sometimes fourth subcultures were necessary.

After confluency was reached in the third or fourth subcultures, the bone marrow-derived cells exhibited relatively uniform spindle-shaped morphology and were positive for STRO-1, a mesenchymal cell marker (data not show). The bone marrow-derived cells were harvested and suspended at $4.0 \times 10^{5}$ cells $/ \mathrm{ml}$ in a spheroid-formation medium composed of DMEM low-glucose (Gibco) supplemented with 10\% regular fetal bovine serum (Biowest) and 1.0\% penicillin-streptomycin solution (Gibco). To form spheroids, the cell suspensions $\left(4.0 \times 10^{4}\right.$ cells/ $\left.0.1 \mathrm{ml}\right)$ were seeded into each well of 96-well U-shaped plates (Sumitomo Bakelite Co., LTD., Tokyo, Japan) and cultured with spheroid-formation medium at $37^{\circ} \mathrm{C}$ in humid air with $5 \% \mathrm{CO}_{2}$ for $2-4$ days. Thus, each of the 96 wells formed a single spheroid. 
The bone marrow-derived cell spheroids were then applied by the 3D bioprinting robot system, Regenova (Cyfuse Biomedical K.K., Tokyo, Japan) to biofabricate the structures. The Regenova picked up the spheroid from each of the 96 wells and inserted each onto a 9x9 microneedle array (approximately $5 \times 5 \mathrm{~mm}$, Fig. 1A). After the spheroids were fully assembled on the surface of the microneedle array, more spheroids were applied to the first layer. For this study, we assembled the sheroids into three layers (approximately 1-mm high, Fig. 1B) on the microneedle array. To induce self-organization, the assembled spheroids were perfusion cultured with spheroid-formation medium at $37^{\circ} \mathrm{C}$ in humid air with $5 \% \mathrm{CO}_{2}$ for 7 days. After the perfusion culture, the microneedle array was removed from the self-organized structure. The bone marrow-derived cell structures, which were biofabricated from 234 spheroids, were approximately 3-mm square and 1-mm high (Fig. 1C).

\section{Transplantation of Biofabricated Bone Marrow-derived Cell Structures}

Two weeks after the last radiation treatment, the recipients were anesthetized with both pentobarbital sodium solution and inhalation of 2-3\% sevoflurane (Mylan Inc., Osaka, Japan). The irradiated bladders were exposed (Fig. 1D), and an approximately 5-mm incision was made on the anterior wall (Fig. 1E). The biofabricated structure was transplanted into the incision ( $\mathrm{n}=10$ rats) and briefly fixed with 7-0 silk suture (Fig. 1F). The transplanted region was covered with absorbable hemostat (Surgicel ${ }^{\circledR}$, Johnson and Johnson K.K., Tokyo, Japan) (Fig. 1G) to avoid the transfer of the transplanted structures to other tissues surrounding the bladder, such as adipose tissues. Finally, the bladders were returned to the pelvic cavity. Control rats were similarly treated except that no structure was inserted into the 5-mm incision (sham-structure controls, $\mathrm{n}=12$ ). The incision was closed with 7-0 suture and covered 
with absorbable hemostat before returning the bladder to the pelvic cavity. All of the structure-transplanted and sham-structure control rats received immunosuppression treatments (described above) every week and were maintained for another 2 or 4 weeks.

\section{Cystometric Investigation}

Two and four weeks after the biofabricated-structure transplantation ( 2 weeks, $n=6 ; 4$ weeks, $n=4$ ) or sham-structure surgery ( $n=6$ in each period), we performed cystometric investigations as previously described $(1,2)$. Briefly, two days prior to the cystometric investigations, a polyethylene catheter was inserted in the bladders. Cystometric investigations were performed on unanesthetized, unrestricted rats placed in each metabolic cage for approximately $30 \mathrm{~min}$. Saline at room temperature was instilled into the bladder through the catheter at a rate of $10 \mathrm{ml} / \mathrm{h}$. Bladder contractions and micturition volume were simultaneously recorded continuously on a pen oscillograph. The following cystometric parameters were measured: basal, threshold, and micturition pressures $\left(\mathrm{cmH}_{2} \mathrm{O}\right)$, voiding interval (minutes), and micturition volume $(\mathrm{ml})$. Residual volume $(\mathrm{ml})$ was calculated by subtracting the micturition volume from the saline infusion volume.

After the cystometric investigations, we harvested the bladders for histological and immunohistochemical investigations (described below). When there was significant adhesion of adipose tissues, we harvested the bladders with the attached adipose tissues to avoid damage by attempts to remove the adherent tissue. 
The trimmed urinary bladders were fixed, embedded in paraffin, and cut in 5- $\mu \mathrm{m}$ thick serial sections. Histological and immunohistochemical investigations were performed according to our previous studies $(1,2)$. For histological investigations, the sections were stained with hematoxylin and eosin (HE), Masson trichrome, enzyme-labeled acetylcholinesterase antibody (Medical \& Biological Laboratories Co., Ltd., Nagoya, Japan), or picrosirius red.

For immunohistochemical investigations the sections were stained with GFP-antibody (1:500, mouse monoclonal, Lifespan Biosciences, Inc., Seattle, WA, USA) to detect the bone marrow-derived cells that composed the biofabricated structures. The GFP-antibody was detected by secondary antibody consisting of donkey anti-mouse IgG conjugated with Alexa fluor 488 (1:250, Molecular Probes, Eugene, OR, USA). The GFP-antibody stained sections were then double-stained with antibody for alpha-smooth muscle actin (SMA, 1:100, mouse monoclonal, Progen Biotechnik GmbH, Heidelberg, Germany) as a marker for smooth muscle cells, or calcitonin gene-related peptide (CGRP, 1:500, guinea pig polyclonal, Progen Biotechnik GmbH) as a marker for afferent nerve cells. These were detected by secondary antibody consisting of donkey anti-mouse or anti-guinea pig IgG conjugated with Alexa fluor 594 (1:250, Molecular Probes, respectively).

Alternatively, other sections were stained with SMA-antibody and detected with secondary antibody consisting of donkey anti-mouse IgG conjugated with Alexa fluor 594 (1:250, Molecular Probes). The SMA-antibody stained sections were double-stained with antibody for collagen prolyl 4-hydroxylase beta (P4HB, 1:50, mouse monoclonal, Novus Biologicals, Inc.), an essential enzyme in the synthesis of all collagens, or hypoxia induced 
factor $1 \alpha(\operatorname{HIF} 1 \alpha)$ antibody (1:50, rabbit polyclonal, Proteintech Group, Inc., Rosemont, IL, USA), a cellular mediator in the responses to hypoxia. The anti-P4HB and anti-HIF1 $\alpha$ antibodies were detected by secondary antibodies consisting of donkey anti-mouse or anti-rabbit IgG conjugated with Alexa fluor 594 (1:250, Molecular Probes, respectively). The immunofluorescent sections were counterstained for nuclei with 4', 6-diamidino-2-phenylindole dihydrochloride (DAPI, $5 \mu \mathrm{g} / \mathrm{ml}$, Molecular Probes).

\section{Statistical Analysis}

Results were expressed as means \pm standard deviations. Statistical differences were determined using the Excel ${ }^{\circledR}$ Statistics program (Esumi Co., Ltd. Tokyo, Japan). Comparisons were made by non-repeated measures analysis of variance (ANOVA). P-values less than 0.05 were considered statistically significant. 


\section{Results}

Biofabricated Bone Marrow-derived cell structures

Biofabricated structures that were not used for transplantation were examined histologically. There were no disorganized defects at center of the structures (Fig. 2A). Within the structures, the assembled spheroids (Fig. 2B) became self-organized through contacts with proliferating cells within and between the spheroids (Fig. 2C) and/or the extracellular matrix secreted from the cells (Fig. 2D). Prior to transplantation, the cells within the structures were positive for GFP-antibody, but negative for SMA- or CGRP-antibody (data not show).

Survival of Biofabricated Bone Marrow-derived Cell Structures Transplanted into Irradiated Urinary Bladders

Two weeks after biofabricated-structure transplantation and sham-structure control surgery, the control bladders did not show any recovery of the incision made on the anterior bladder wall (Fig. 3A). Furthermore, the injured regions had numerous inflammatory cells with a macronucleus and/or eosin-staining cytoplasm, similar to macrophages and/or acidophilic leukocytes (Fig. 3B). In addition, the smooth muscle layers were thin and sparsely distributed (Fig. 3C). In contrast, the biofabricated structures were readily recognized at the transplanted regions on the anterior bladder wall (Fig. 3D). The transplanted structures survived within the recipient tissues, and there were few inflammatory cells within the bladder tissues near the transplanted regions (Fig. 3E). Within the bladder 
tissues near the transplanted structures, distinct smooth muscle layers were present (Fig. 3F). Further, there were blood vessels within the border between the transplanted structures and the recipient tissues (Fig. 3G). The smooth muscle cells within the walls of the blood vessels were negative for GFP-antibody (Fig. 3H). Thus, the blood vessels originated from the recipient tissues and extended into the transplanted structures. Surrounding the extended blood vessels, the bone marrow-derived cells that composed the structures, which were simultaneously positive for GFP (Fig. 3H) and SMA (Fig. 3I) antibody, had differentiated into smooth muscle cells (Fig. 3J). While the double-positive differentiated smooth muscle cells were widely distributed, they did not form smooth muscle clusters (Fig. 3J).

Four weeks after the sham-structure control surgery, the incision had only slightly recovered compared to the control bladders at 2 weeks after surgery (Fig. 4A). The control operated regions had numerous inflammatory cells (Fig. 4B), and smooth muscle layers were disorganized (Fig. 4C). In contrast, the transplanted structures were easily identified (Fig. 4D) and were well integrated into the recipient bladder walls (Fig. 4E). The recipient bladder wall near the transplanted regions had distinct smooth muscle layers (Fig. 4F) that were not observed in the sham-structure control operated regions (Fig. 4C) at the same time after surgery. In addition, the reconstructed smooth muscle layers were similar to non-irradiated normal bladders (1). Within the transplanted structures, the differentiated smooth muscle cells that surrounded the blood vessels originating from the recipient tissues formed smooth muscle clusters (Fig. 4G-I). Also, there were small smooth muscle clusters composed of differentiated smooth muscle cells at the outside of the transplanted structures (Fig. 4J-L). 
Histological Changes of Recipient Bladder Tissues

In immunohistochemical and histological investigations, we mainly observed bladder tissues near the border between the recipient tissues and the transplanted structures or the incised regions of the sham-structure control bladders. Two weeks after the control surgery, acetylcholinesterase-positive cells (Fig. 5A) and CGRP-positive afferent nerve cells (Fig. 5B) were only sparsely present. At 2 weeks after structure transplantation, some acetylcholinesterase-positive cells were present (Fig. 5C). In contrast, the content and distribution of CGRP-positive afferent nerve cells were similar to the control bladder tissues at same time after transplantation (Fig. 5D).

Four weeks after the sham-structure control surgery, acetylcholinesterase-positive cells were still not apparent (Fig. 5E), and there were fewer CGRP-positive afferent nerve cells compared to the recipient control bladders at 2 weeks after sham-structure surgery (Fig. 5F). However, at 4 weeks after the structure transplantation, the recipient bladder tissues had numerous acetylcholinesterase- (Fig. 5G) and CGRP-positive cells (Fig. 5H), significantly more than was evident in the transplanted recipient bladders at 2 weeks after surgery. None of the CGRP-positive cells were positive for GFP in either the transplanted structures or the recipient bladder tissues at either 2 or 4 weeks after surgery (data not shown).

By two weeks after the sham-structure control surgery, collagen fibers had invaded the spaces of the extracellular matrix that contained damaged smooth muscle layers (Fig. 6A). The cells in the collagen fiber areas expressed the fibrosis marker P4HB (Fig. 6B). In addition, the control bladder tissues had numerous HIF1 $\alpha$-positive cells, markers of hypoxia (Fig. 6C). In contrast, at two weeks after the structure transplantation, the collagen fibers in 
the recipient bladder tissues were distributed among the reconstructed smooth muscle clusters (Fig. 6D). Among the smooth muscle clusters, there were a few P4HB- (Fig. 6E) and HIF1 $\alpha$-positive cells (Fig. 6F); however, the numbers of both P4HB- and HIF1 $\alpha$-positive cells were lower compared to the control bladder tissues at the same time after surgery.

At 4 weeks after the sham-structure control surgery, the collagen fibers were greatly expanded and occupied the extracellular spaces in the absence of smooth muscle layers (Fig. 6G). The regions with a few smooth muscle layers had numerous P4HB- (Fig. 6H) and HIF1 $\alpha$-positive cells (Fig. 6I). In contrast, the collagen fibers in the structure-transplanted recipient bladder tissues were integrated among the smooth muscle clusters (Fig. 6J) and were distributed similar to the transplanted recipient bladder tissues at 2 weeks after surgery (Fig. 6D). At 4 weeks after structure transplantation, the recipient bladder tissues also had a few P4HB- (Fig. 6K) and HIF1 $\alpha$-positive cells (Fig. 6L) within the collagen fibers, but the numbers of these cells were lower compared to control bladder tissues at the same time after surgery (Fig. 6H and I, respectively).

\section{Recovery of Urinary Bladder Functions}

In cystometric investigations at 2 weeks after either sham-structure control surgery or structure transplantation, the micturition patterns were similar for the two groups (data not shown). At 4 weeks after the control surgery, the rats exhibited significant urinary frequency symptoms that included irregular voiding intervals of less than 5 min with micturition volumes of less than $1 \mathrm{ml}$ (Fig. 7A). However, the structure transplanted rats showed regular micturitions with voiding intervals of about 5 min and micturition volumes of about $1 \mathrm{ml}$ (Fig. 7B). Thus, for rats with radiation-injured urinary bladders that received biofabricated 
structure transplants, the urinary frequency symptoms at 4 weeks after surgery were improved and/or mitigated compared to the sham-structure control rats at same time after surgery.

Each micturition parameter was estimated from the cystometric charts. At 2 weeks after sham-structure control surgery or structure transplantation, the basal, threshold, and micturition pressures, and the voiding interval and micturition volume were not significantly different between the control and the structure transplantation group (Fig. 8A-E). However, residual volume of the structure-transplanted rats $(0.02 \pm 0.01 \mathrm{ml})$ was significantly lower than controls $(0.08 \pm 0.02 \mathrm{ml}, \mathrm{P}<0.05$, Fig. 8F). Thus, micturition efficiency of the structure-transplanted rats was improved compared to the control rats.

At 4 weeks after sham-structure control surgery, the basal (Fig. 8A), threshold (Fig. 8B), and micturition pressures (Fig. 8C) and the residual volume (Fig. 8F) did not change from these values at 2 weeks after sham-structure control surgery. However, the voiding interval, $3.40 \pm 0.43 \mathrm{~min}$, and micturition volume, $0.55 \pm 0.09 \mathrm{ml}$, of the control rats at 4 weeks after control surgery were significantly lower than the control rats at 2 weeks after control surgery, 6.52 $\pm 0.81 \mathrm{~min}(\mathrm{P}<0.01$, Fig. 8D) and $1.04 \pm 0.14 \mathrm{ml}(\mathrm{P}<0.05$, Fig. 8E), respectively. In contrast, at 4 weeks after structure transplantation, none of the micturition parameters changed from the values at 2 weeks after structure transplantation (Fig 8).

At 4 weeks after sham-structure control surgery or structure transplantation, the basal, threshold, and micturition pressures were not significantly different between the sham-structure control and the structure transplantation group (Fig. 8A-C). However, the voiding interval (4.93 $\pm 0.63 \mathrm{~min}$, Fig. $8 \mathrm{D})$ and micturition volume $(0.83 \pm 0.12 \mathrm{ml}$, Fig. $8 \mathrm{E})$ of 
structure transplanted rats at 4 weeks after surgery were significantly higher than the control rats at the same time after surgery $(\mathrm{P}<0.01$, and $\mathrm{P}<0.05$, respectively). In addition, the residual volume of the structure transplanted rats at 4 weeks after surgery, $0.02 \pm 0.01 \mathrm{ml}$, was significantly lower than that of the sham-structure control rats at the same time after surgery, $0.05 \pm 0.01 \mathrm{ml}(\mathrm{P}<0.05$, Fig. 8F). These micturition parameters in the structure-transplanted bladders were similar to the non-irradiated normal bladders (1). These results showed that the biofabricated structure transplanted rats partially inhibited the decrease of both storage function and micturition efficiency that occurred in the radiation-injured bladders. 


\section{Discussion}

We have vigorously investigated methods to assist in the structural and functional recovery of the lower urinary tract, which is mainly composed of the bladder and urethra. To achieve our goals, we have applied tissue engineering methodology that utilizes a combination of cells, (bio-)materials, and biochemical factors based on the microenvironment within the target tissues and organs of the recipients $(25,26)$. Recently, biofabrications enabled by 3D bioprinters have been reported as novel biotechnologies (3). Thus, we have now incorporated this novel biotechnology into our tissue engineering methodology. We call this methodology “next generation tissue engineering”.

With the 3D bioprinting robot system Regenova, we biofabricated self-organizing tissue-like structures composed of bone marrow-derived cells. The biofabricated structures have some advantages that are not present in either the cell-injection or cell-sheet methods. First, the structures have sufficient thickness and strength to facilitate handling for transplantation. Second, the structures can be directly transplanted into the recipient tissues. Third, the 3D-conformation provided cell-to-cell contacts that imitate the naturally occurring tissues and promote self-organization. Finally, the structures are likely to have higher biocompatibility compared to artificial materials.

While investigation of the biofabricated structure effects on the recipient tissues was not a principal focus of this study, paracrine effects on the microenvironment of the adjacent recipient tissues are to be expected (2). Therefore, the histological changes at the interface between the transplanted structures and the recipient tissues are important. The most important results are that the transplanted structures survived within the recipient tissues, and 
blood vessels grew into them from the adjacent recipient tissues. Surrounding the extended blood vessels, the bone marrow-derived cells in the structures differentiated into smooth muscle cells. At 4 weeks after transplantation, they formed clusters of smooth muscle cells at the surrounding extended blood vessels and the outside edges of the transplanted structures. Within the transplanted structures, HIF1 $\alpha$-positive cells, which indicate a hypoxic state, were sparsely distributed (data not shown). Thus, the microenvironment surrounding the blood vessels that extended from recipient tissues supported the differentiation of smooth muscle cells and formation of smooth muscle clusters, which could have differentiated from the bone marrow-derived cells.

Signal pathways involving hypoxia-dependent HIF $1 \alpha$ expression are reported to give rise to the fibrosis in damaged urinary bladders (27-29). However, within the recipient tissues of the structure-transplanted bladders at both 2- and 4-weeks after surgery, there were fewer hypoxia marker HIF1 $\alpha$-positive cells compared to the sham-structure controls. In addition, the structure transplanted bladders did not develop significant fibrosis or contain many P4HB-positive cells. The presence of the biofabricated structures and the associated ingrowth of blood vessels from the surrounding tissues may have created an optimal microenvironment and reduced or eliminated the wound-associated hypoxia. Consequently, it is likely that the HIF $1 \alpha$ pathways were only transiently and/or minimally activated, and therefore activation of extracellular matrix collagen formation via the P4HB pathway was limited. This could account for the absence of significant fibrosis in the structure-transplanted bladders. These histological findings in the structure transplanted-bladders were similar to ones in non-irradiated normal bladders (1) (Supplemental Figure). 
Additionally, we showed that transplantation of the bone marrow-derived cell structures into the irradiated bladders induced recovery of bladder functionality, as occurred in our previous studies $(1,2)$. At 4 weeks after implantation, the structure-transplanted rats did not show significant urinary frequency symptoms. The voiding intervals and volumes were higher compared to the control rats. In addition, residual volume of the rats with structure transplants was lower than that of the control rats at both 2 and 4 weeks after implantation. The transplantation of biofabricated bone marrow-derived cell structures improved both storage function and micturition efficiency, both of which were restored to the levels in non-irradiated normal bladders (1) (Supplemental Figure). Additionally, these results suggest that the transplantation structures might inhibit progressive irradiation damage. It is likely that the improvements or inhibitions were related to the mitigation of irradiation-induced urinary frequency symptoms.

Aside from the previously described advantages of biofabricated structures over single cell direct-injection and cell sheets patch-transplantation methods, the biofabricated structures deliver many more cells to the target site. Construction of the structure inserts 245 spheroids in/on the 9x9 microneedle array. Each spheroid was formed with 4x10 4 cells. Thus, the approximate number of cells in each biofabricated structure was $1 \times 10^{7}$ cells (245 spheroids $\times 4 \times 10^{4}$ cells/spheroid $=\sim 1 \times 10^{7}$ cells). This is about 100 -times more cells than used in either the cell-injection or cell-sheet methods. At 2 weeks after transplantation, which was half of the period of our previous studies $(1,2)$, the bladder functions of the structure-transplanted rats, except for residual volume, was not different from the sham-structure controls. Thus, the recovery at two weeks after transplantation does not appear to be strictly related to the number of implanted bone marrow-derived cells. This issue 
has been discussed among researchers and clinicians. Our data suggests that reconstruction of functional tissues needs a certain recovery period, at least 4 weeks for the experimental model here, for cell replication, differentiation, and tissue organization. During that recovery period, the transplanted biofabricated structures were able to substitute and replace the injured tissues, thus providing regenerative effects that were not evident in the direct-injection and cell sheet methods.

To develop the utilization of the biofabricated structures, further investigations are warranted. Scale-up of the biofabricated structures are needed for large animal experiments and treatments for human lower urinary tract symptoms. To conduct the scale-up, we need to establish large-scale cell culture systems and efficient spheroid formation techniques. Another limitation that we encountered was that after removal of the microneedle array, the biofabricated structure shrank from the original 5-mm square to about 3-mm square. We attribute this shrinkage to the DMEM low-glucose culture medium. Therefore, we need to develop a suitable culture medium that inhibits shrinkage of the biofabricated structures.

In conclusion, we used a 3D bioprinting robot system to biofabricate structures composed of bone marrow-derived cells. The structures, when transplanted into the irradiation wounded rat urinary bladders, survived, and blood vessels invaded them from the adjacent recipient tissues. The bone marrow-derived cells constructing the structures differentiated into smooth muscle cells and formed smooth muscle clusters. While the cells did not differentiate into nerve cells, regenerated nerve cells were present within the recipient bladder tissues. The structure-transplanted bladder tissues did not develop significant fibrosis associated with HIF1 $\alpha$ - and P4HB-positive cells. At 4 weeks after structure transplantation, 
the rats had improved urinary frequency symptoms and decreased residual volume. Therefore, biofabricated structures have great potential to treat patients with serious lower urinary tract symptoms due to damaged bladders. 


\section{Acknowledgments}

This study was supported by a Grant-in-Aid for Scientific Research (C) from Japan Society for the Promotion of Science (15K10622). The authors thank Ms. Nanami Hatakeyama for her great contributions in constructing the biofabricated structures.

\section{Disclosure Statements}

No competing financial interests exist. 


\section{References}

1. Imamura, T., Ishizuka, O., Lei, Z., Hida, S., Sudha, G.S., Kato, H., et al. Bone marrow-derived cells implanted into radiation-injured urinary bladders reconstruct functional bladder tissues in rats. Tissue Eng Part A 18, 1698, 2012.

2. Imamura, T., Ogawa, T., Minagawa, T., Yokoyama, H., Nakazawa, M., Nishizawa, O., et al. Engineered bone marrow-derived cell sheets restore structure and function of radiation-injured rat urinary bladders. Tissue Eng Part A 21, 1600, 2015.

3. Bajaj, P., Schweller, R.M., Khademhosseini, A., West, J.L., and Bashir, R. 3D biofabrication strategies for tissue engineering and regenerative medicine. Annu Rev Biomed Eng 16, 247, 2014.

4. Kim, J.J., Hou, L., and Huang, N.F. Vascularization of three-dimensional engineered tissues for regenerative medicine applications. Acta Biomater 41, 17, 2016.

5. Boland, T., Xu, T., Damon, B., and Cui, X. Application of inkjet printing to tissue engineering. Biotechnol J 1, 910, 2006.

6. Lorber, B., Hsiao, W.K., Hutchings, I.M., and Martin, K.R. Adult rat retinal ganglion cells and glia can be printed by piezoelectric inkjet printing. Biofabrication 6, 015001, 2014.

7. Nganga, S., Moritz, N., Kolakovic, R., Jakobsson, K., Nyman, J.O., Borgogna, M., et al. Inkjet printing of Chitlac-nanosilver--a method to create functional coatings for non-metallic bone implants. Biofabrication 6, 041001, 2014.

8. Tse, C., Whiteley, R., Yu, T., Stringer, J., MacNeil, S., Haycock, J.W., et al. Inkjet printing Schwann cells and neuronal analogue NG108-15 cells. Biofabrication 8, 015017, 2016.

9. Xu, T., Jin, J., Gregory, C., Hickman, J.J., and Boland, T. Inkjet printing of viable mammalian cells. Biomaterials 26, 93, 2005.

10. Yamaguchi, S., Ueno, A., Akiyama, Y., and Morishima, K. Cell patterning through inkjet printing of one cell per droplet. Biofabrication 4, 045005, 2012.

11. Hanson Shepherd, J.N., Parker, S.T., Shepherd, R.F., Gillette, M.U., Lewis, J.A., and Nuzzo, R.G. 3D Microperiodic Hydrogel Scaffolds for Robust Neuronal Cultures. Adv Funct Mater 21, 47, 2011.

12. Sakai, S., Ohi, H., Hotta, T., Kamei, H., and Taya, M. Differentiation potential of human adipose stem cells bioprinted with hyaluronic acid/gelatin-based bioink through 
microextrusion and visible light-initiated crosslinking. Biopolymers 2017. DOI: 10.1088/bit.23080

13. Wenz, A., Borchers, K., Tovar, G.E.M., and Kluger, P. Bone matrix production in hydroxyapatite-modified hydrogels suitable for bone bioprinting. Biofabrication 2017. DOI: 10.1088/1758-5090/aa91ec

14. Zhao, Y., Li, Y., Mao, S., Sun, W., and Yao, R. The influence of printing parameters on cell survival rate and printability in microextrusion-based 3D cell printing technology. Biofabrication 7, 045002, 2015.

15. Bourget, J.M., Kerouredan, O., Medina, M., Remy, M., Thebaud, N.B., Bareille, R., et al. Patterning of endothelial cells and mesenchymal stem cells by laser-assisted bioprinting to study cell migration. Biomed Res Int 2016, 3569843, 2016.

16. Catros, S., Fricain, J.C., Guillotin, B., Pippenger, B., Bareille, R., Remy, M., et al. Laser-assisted bioprinting for creating on-demand patterns of human osteoprogenitor cells and nano-hydroxyapatite. Biofabrication 3, 025001, 2011.

17. Desrus, H., Chassagne, B., Moizan, F., Devillard, R., Petit, S., Kling, R., et al. Effective parameters for film-free femtosecond laser assisted bioprinting. Appl Opt 55, 3879, 2016.

18. Devillard, R., Pages, E., Correa, M.M., Keriquel, V., Remy, M., Kalisky, J., et al. Cell patterning by laser-assisted bioprinting. Methods Cell Biol 119, 159, 2014.

19. Gruene, M., Unger, C., Koch, L., Deiwick, A., and Chichkov, B. Dispensing pico to nanolitre of a natural hydrogel by laser-assisted bioprinting. Biomed Eng Online 10, 19, 2011.

20. Guillotin, B., Souquet, A., Catros, S., Duocastella, M., Pippenger, B., Bellance, S., et al. Laser assisted bioprinting of engineered tissue with high cell density and microscale organization. Biomaterials 31, 7250, 2010.

21. Keriquel, V., Oliveira, H., Remy, M., Ziane, S., Delmond, S., Rousseau, B., et al. In situ printing of mesenchymal stromal cells, by laser-assisted bioprinting, for in vivo bone regeneration applications. Sci Rep 7, 1778, 2017.

22. Michael, S., Sorg, H., Peck, C.T., Koch, L., Deiwick, A., Chichkov, B., et al. Tissue engineered skin substitutes created by laser-assisted bioprinting form skin-like structures in the dorsal skin fold chamber in mice. PLoS One 8, e57741, 2013. 
23. Moldovan, N.I., Hibino, N., and Nakayama, K. Principles of the Kenzan method for robotic cell spheroid-based three-dimensional bioprinting. Tissue Eng Part B: Rev 23, 237, 2017.

24. Itoh, M., Nakayama, K., Noguchi, R., Kamohara, K., Furukawa, K., Uchihashi, K., et al. Correction: Scaffold-free tubular tissues created by a bio-3d printer undergo remodeling and endothelialization when implanted in rat aortae. PLoS One 10, e0145971, 2015.

25. Imamura, T., Ishizuka, O., Yamamoto, T., Gotoh, M., and Nishizawa, O. Bone marrow-derived cells implanted into freeze-injured urinary bladders reconstruct functional smooth muscle layers. Low Urin Tract Symptoms 2, 1, 2010.

26. Imamura, T., Yamamoto, T., Ishizuka, O., Gotoh, M., and Nishizawa, O. The microenvironment of freeze-injured mouse urinary bladders enables successful tissue engineering. Tissue Eng Part A 15, 3367, 2009.

27. Ekman, M., Uvelius, B., Albinsson, S., and Sward, K. HIF-mediated metabolic switching in bladder outlet obstruction mitigates the relaxing effect of mitochondrial inhibition. Lab Invest 94, 557, 2014.

28. Iguchi, N., Donmez, M.I., Malykhina, A.P., Carrasco, A., Jr., and Wilcox, D.T. Preventative effects of a HIF inhibitor, 17-DMAG, on partial bladder outlet obstruction-induced bladder dysfunction. Am J Physiol Renal Physiol, 313, F1149, 2017.

29. Wiafe, B., Adesida, A., Churchill, T., Adewuyi, E.E., Li, Z., and Metcalfe, P. Hypoxia-increased expression of genes involved in inflammation, dedifferentiation, pro-fibrosis, and extracellular matrix remodeling of human bladder smooth muscle cells. In Vitro Cell Dev Biol Anim 53, 58, 2017.

Address correspondence to:

Tetsuya Imamura, PhD.

Department of Urology

Shinshu University School of Medicine

3-1-1 Asahi, Matsumoto

Nagano, 390-8621

JAPAN

E-mail: imatetu@shinshu-u.ac.jp 


\section{Figure Legends}

FIGURE 1. Biofabrication and transplantation of the bone marrow-derived structures. (A) Bone marrow-derived cell spheroids, which were formed from $4.0 \times 10^{4}$ cells, were assembled into a 9x9 microneedle array (approximately 5x5 mm when viewed from above). (B) Accumulated bone marrow-derived cell spheroids (3-layers, approximately $1 \mathrm{~mm}$ when viewed from the side). (C) At 7 days of perfusion culture, and after removing the microneedle array, the biofabricated bone marrow-derived structure was approximately 3x3 mm and 1-mm high. (D) At 2 weeks after the last radiation treatment, the irradiated bladder of the recipient rat was exposed. (E) An approximately 5-mm incision (double-headed arrow) was made on the anterior wall of the irradiated bladders. (F) The biofabricated structure (asterisk) was transplanted into the incision of the irradiated anterior bladder wall, and briefly fixed with 7-0 silk suture. (G) The transplantation region was covered with absorbable hemostat (arrowhead).

FIGURE 2. Biofabricated bone marrow-derived cell structures. (A) There were no disorganized defects at the center of the structures. HE stain. (B and C) The assembled spheroids (B: asterisks, Masson trichrome stain) contacted each other through proliferating cells (C: arrows, HE stain) and became self-organized to form the structures. (D) In this serial section from the same region, the assembled spheroids contacted each other through extracellular matrix secreted from the bone marrow-derived cells (arrowheads). Masson trichrome stain.

FIGURE 3. Histological investigations of urinary bladders at 2 weeks after sham-structure control surgery $(n=6)$ and transplantation of the biofabricated structure $(n=6)$. (A-C) Shamstructure control bladders. (A) There was no apparent healing of the wound for the incision 
made on the anterior bladder wall (large arrowheads). (B) The wounded regions had numerous inflammatory cells (small arrowheads). (C) Within the wounded regions, smooth muscle layers were thin and sparsely distributed (black asterisks). (D-F) Biofabricated structure transplanted bladders. (D) The transplanted biofabricated structure (black arrow) was evident on the anterior bladder wall. (E) The transplanted structure (black arrows) survived, and there were few inflammatory cells within the bladder tissues near the transplanted region. (F) Smooth muscle layers (black asterisks) were reconstructed within the bladder tissues near the transplanted structure (black arrows). White asterisks in A-F: adhesion of adipose tissues that could not be removed. White arrows in D-F: 7-0 silk suture scar. (G) Box in F, the transplanted structure had blood vessels (black arrows). (H) Within the same regions of Masson trichrome stained serial sections, smooth muscle cells, including the blood vessels, were negative for GFPantibody. Thus, the blood vessels within the transplanted structure were extensions of vessels from the recipient tissues (arrows). The bone marrow-derived cells constructing the structures were detected with GFP-antibody (green). (I) The cells in the transplanted structure were positive for SMA-antibody (red). Blood vessels (arrows). (J) The double-positive cells (yellow) were differentiated smooth muscle cells. Blue: nuclei. Blood vessels (arrows).

FIGURE 4. Histological investigations of urinary bladders at 4 weeks after sham-structure control surgery $(n=6)$ and transplantation of the biofabricated structure $(n=4)$. (A-C) Shamstructure control bladders. (A) The incision was not completely healed (large arrowheads). (B) Within the wounded regions, there were numerous inflammatory cells (small arrowheads). (C) The smooth muscle layers within the wounded regions were disorganized. White asterisks in A-C: adhesion of adipose tissue that could not be removed. (D-F) Biofabricated structuretransplanted bladders. (D) The transplanted biofabricated structure (black arrow) was easily 
seen on the anterior bladder wall. The transplanted structure (black arrow) appeared to be well integrated into the recipient bladder wall. (E) The biofabricated structure (black arrows) transplanted into the bladder was histologically intact and had few inflammatory cells. (F) The smooth muscle layers (black asterisks) within the bladder tissues near the transplanted regions were reconstructed, like those in the structure-transplanted bladder tissues at 2 weeks after transplantation. White arrow in B-F: 7-0 silk suture scar. (G-I) Box a in F, within the biofabricated structures transplanted in the irradiated bladders, both GFP- (G: green) and SMApositive (H: red) differentiated smooth muscle cells surrounded the blood vessels (asterisk) that extended from the recipient tissues, and formed smooth muscle clusters (I: yellow). Blue: nuclei. (J-L) Box b in F, near the outside edges of the transplanted structures, both GFP- (J: green) and SMA-positive (K: red) differentiated smooth muscle cells formed clusters (L: yellow). Blue: nuclei.

FIGURE 5. Reconstruction of nerve fibers within recipient bladder tissues near both the incision border of the sham-structure control surgery and the transplanted biofabricated structures. (A and B) Two weeks after the control sham-structure control surgery ( $n=6$ ), the control bladders had few acetylcholinesterase-positive cells (A), while some CGRP-positive afferent nerve cells were present (B: red, white arrowheads). (C and D) Two weeks after structure transplantation $(n=6)$, the recipient bladders had a few acetylcholinesterase-positive cells (C: brown stain, black arrow) and CGRP-positive cells (D: red, white arrowheads). (E and F) Four weeks after the control surgery $(n=6)$, the presence of acetylcholinesterase-positive cells (E) was similar to that at two weeks after surgery, but there were fewer CGRP-positive cells than at two weeks after the surgery (F: red, white arrowhead). ( $G$ and $H$ ) On the other hand, at 4 weeks after structure transplantation $(n=4)$, the recipient bladders had many more 
acetylcholinesterase- (G: brown stain, black arrows) and CGRP-positive cells (H: red, white arrowheads) compared to 2 weeks after structure transplantation. However, none of the CGRPpositive cells in the recipient tissues co-localized with GFP; therefore, the CGRP-positive cells did not differentiate from bone marrow-derived cells at either 2- or 4-weeks after structure transplantation.

FIGURE 6. Disposition of collagen fibers, collagen-associated P4HB-positve cells, and hypoxia-associated HIF $1 \alpha$-positive cells in the recipient bladders. (A) At 2 weeks after the sham-structure control surgery ( $n=6$ ), collagen fibers (asterisks) had invaded the extracellular matrix surrounding the damaged smooth muscle layers. (B) In control bladders, the cells within the collagen fiber areas expressed the fibrosis marker, P4HB (green, arrows). (C) At the same time, control bladders had numerous hypoxia marker HIF1 $\alpha$-positive cells (green, arrowheads). (D) At 2 weeks after the biofabricated structure was transplanted ( $n=6)$, the collagen fibers (asterisks) were distributed among the reconstructed smooth muscle clusters. (E and F) Among the smooth muscle clusters, there were a few P4HB- (E: green, arrows) and HIF1 $\alpha$-positive cells (F: green, arrowheads). (G) At 4 weeks after the control sham surgery ( $n=6)$, the collagen fibers (asterisks) had greatly expanded and there was a large amount of the extracellular matrix in the absence of smooth muscle layers. (H and I) Regions with only few smooth muscle layers had numerous P4HB- (H: green, arrows) and HIF1 $\alpha$-positive cells (I: green, arrowheads). (J) At the same time, structure-transplanted recipient bladders had collagen fibers (asterisks) that were distributed among the smooth muscle clusters, similar to those at 2 weeks after transplantation. (K and L) Four weeks after structure transplantation $(n=4)$, the bladder tissues also had a few P4HB- (K: green, arrows) and HIF1 $\alpha$-positive cells (L: green, white arrowheads) among the smooth muscle clusters. Red: SMA-positive smooth muscle cells. Blue: nuclei. 
FIGURE 7. Micturition patterns of sham-structure control $(n=6)$ and biofabricated structure transplanted rats $(n=4)$ at 4 weeks after surgery. (A) The sham-structure control rats exhibited significant urinary frequency symptoms such as irregular voiding intervals of less than 5 min (upper trace: arrowheads, voiding points) with micturition volumes of less than $1 \mathrm{ml}$ (lower trace) (B) The structure transplanted rats had regular micturitions with voiding intervals of about 5 min (upper trace: arrowheads, voiding points) and micturition volumes of about $1 \mathrm{ml}$ (lower trace).

FIGURE 8. Comparison of micturition parameters between sham-structure control rats ( $\mathrm{n}=6$ in each period) and biofabricated structure transplanted rats ( 2 weeks, $n=6$; 4 weeks, $n=4$ ). At 2 weeks after surgery, there were no differences between the two groups for basal (A), threshold (B), and micturition pressure (C), voiding interval (D), or micturition volume (E). However, the residual volume (F) at 2 weeks after structure transplantation was significantly lower than the controls at same time after surgery. At 4 weeks after sham-structure control surgery, while basal (A), threshold (B) and micturition pressures (C), and residual volume (F) did not change from the values at 2 weeks after surgery, voiding interval (D) and micturition volume (E) were siginificantly lower than the respective values at 2 weeks after surgery. At 4 weeks after structure transplantation, none of the micturition parameters changed from the values at 2 weeks after surgey. At 4 weeks after control surgery or structure transplantation, basal (A), threshold (B), and micturition pressures (C) were not significantly different between the shamstructure control and the structure transplantation group. However, both voiding interval (D) and micturition volume (E) in the structure transplanted rats were significantly higher than these in the control rats. In addition, residual volume $(\mathrm{F})$ of the structure transplanted rats was significantly lower than that of the control rats. White bars: sham-structure control rats. Gray 
bars: biofabricated-structure transplanted rats. ${ }^{*} \mathrm{P}<0.05$, compared to the sham-structure controls within each period; $\uparrow \mathrm{P}<0.05$, $\dagger \dagger \mathrm{P}<0.01$, compared to 2 weeks after operation within each group. 


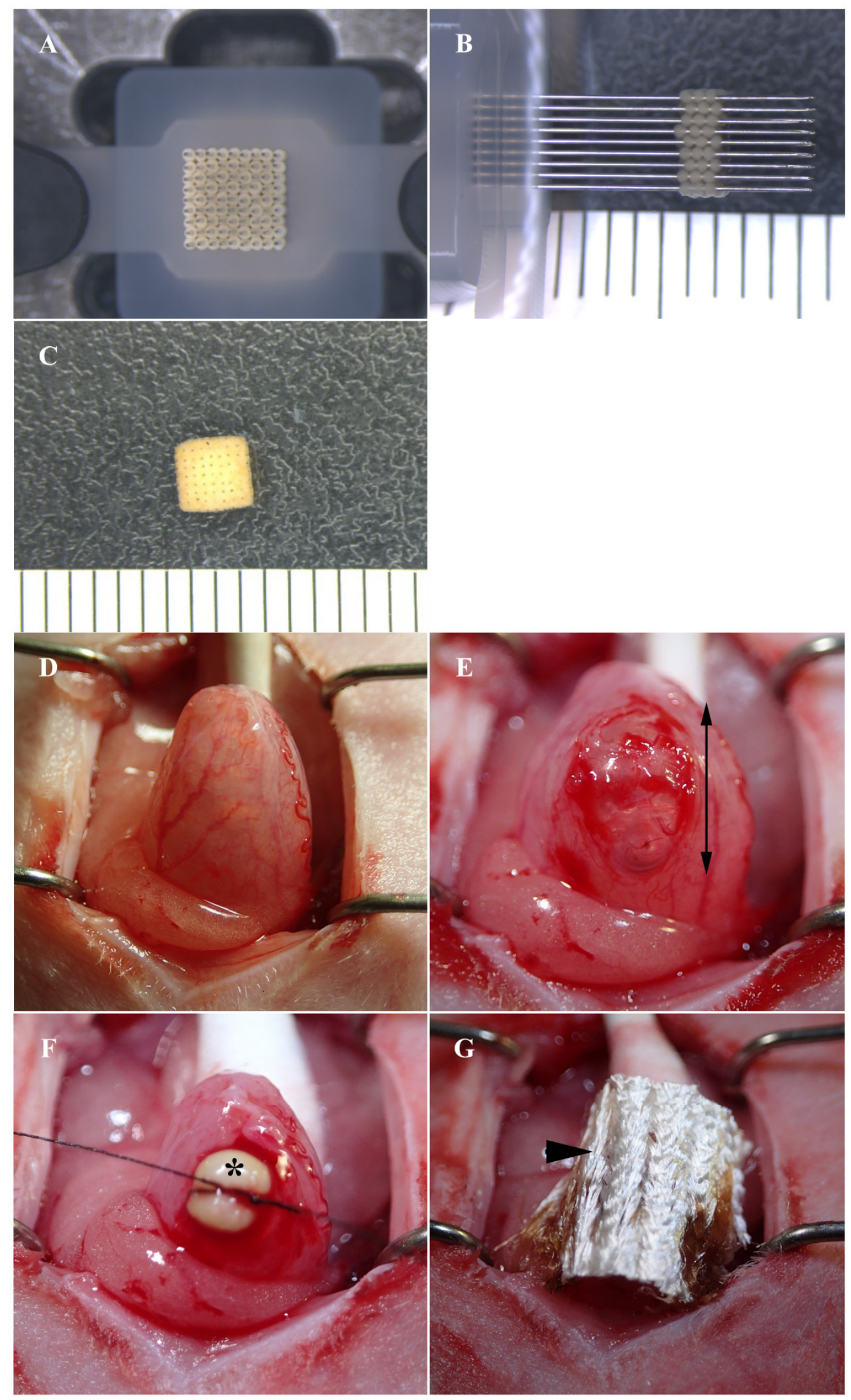

FIG. 1 


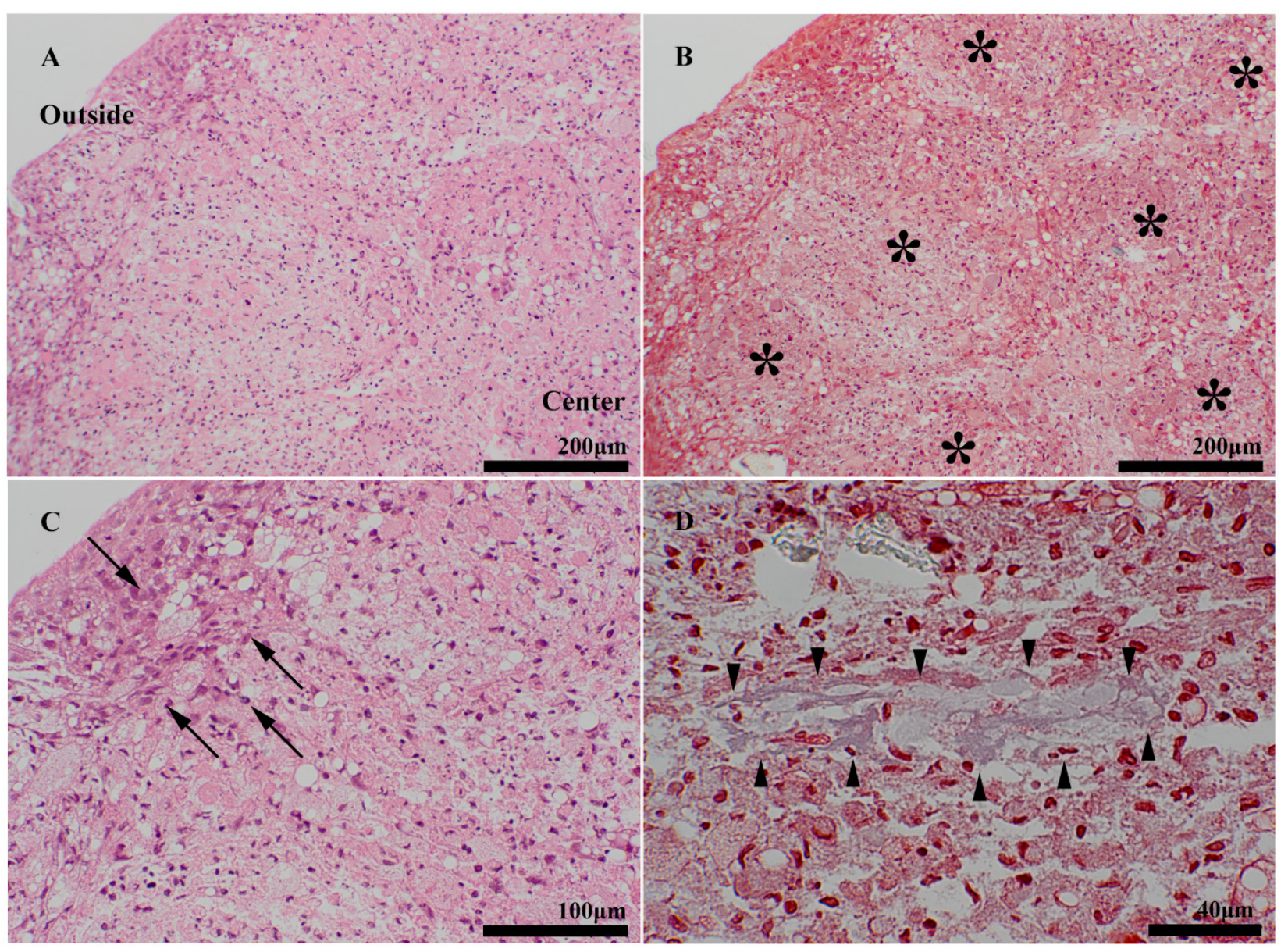

FIG. 2 

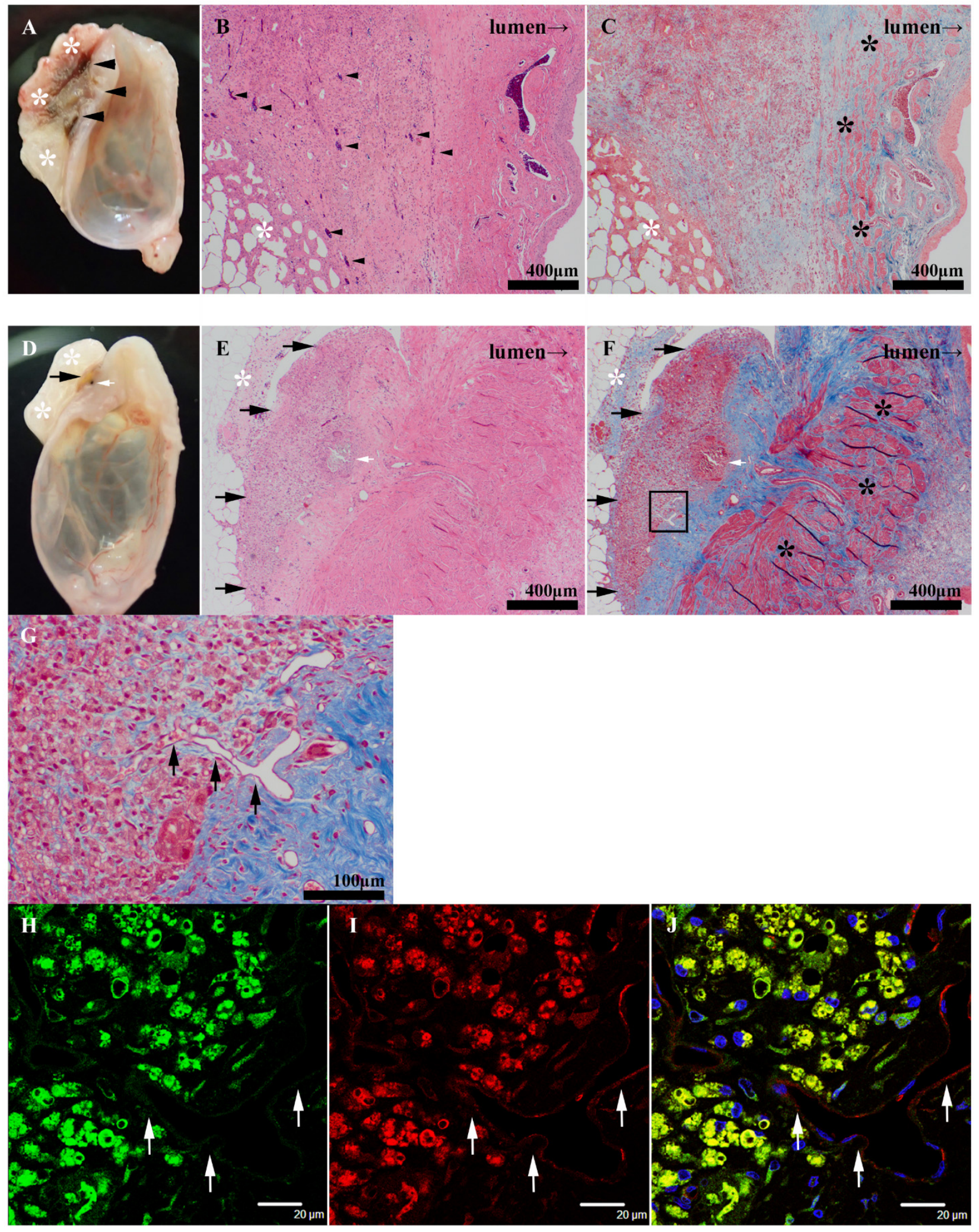

FIG. 3 



FIG. 4 



FIG. 5 
Picrosirius Red Stain
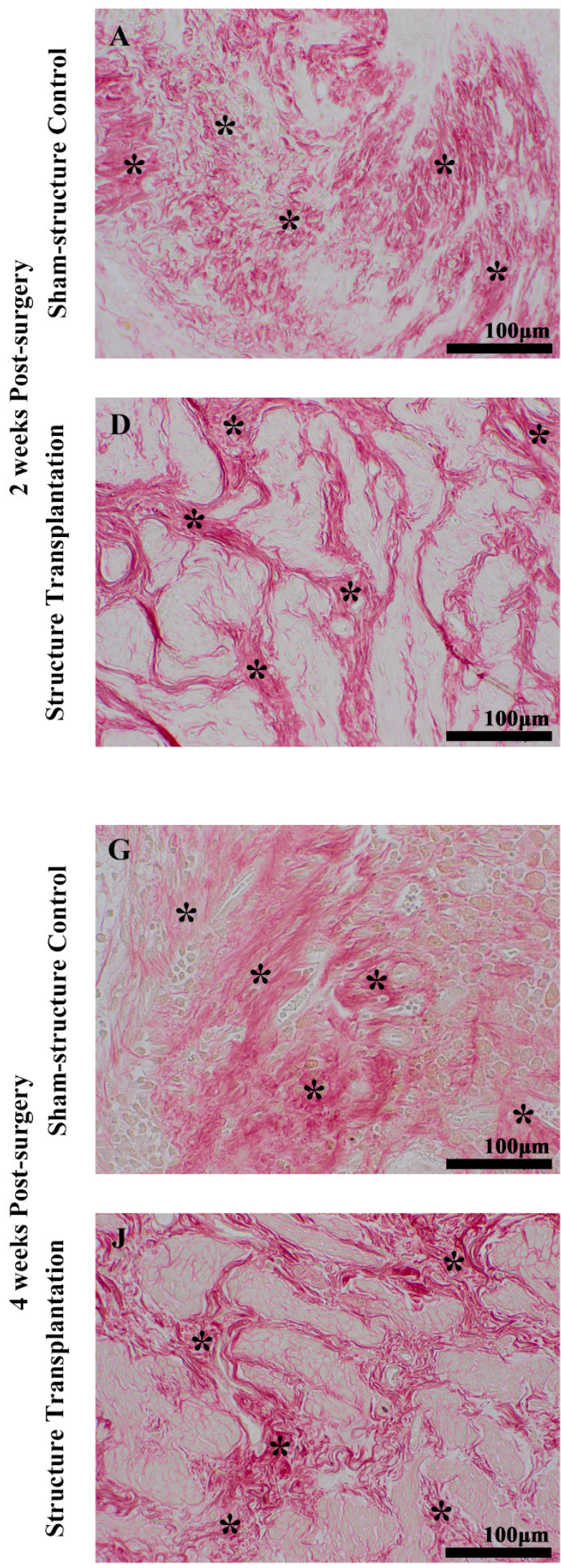

Immunohistochemistry

P4HB / SMA
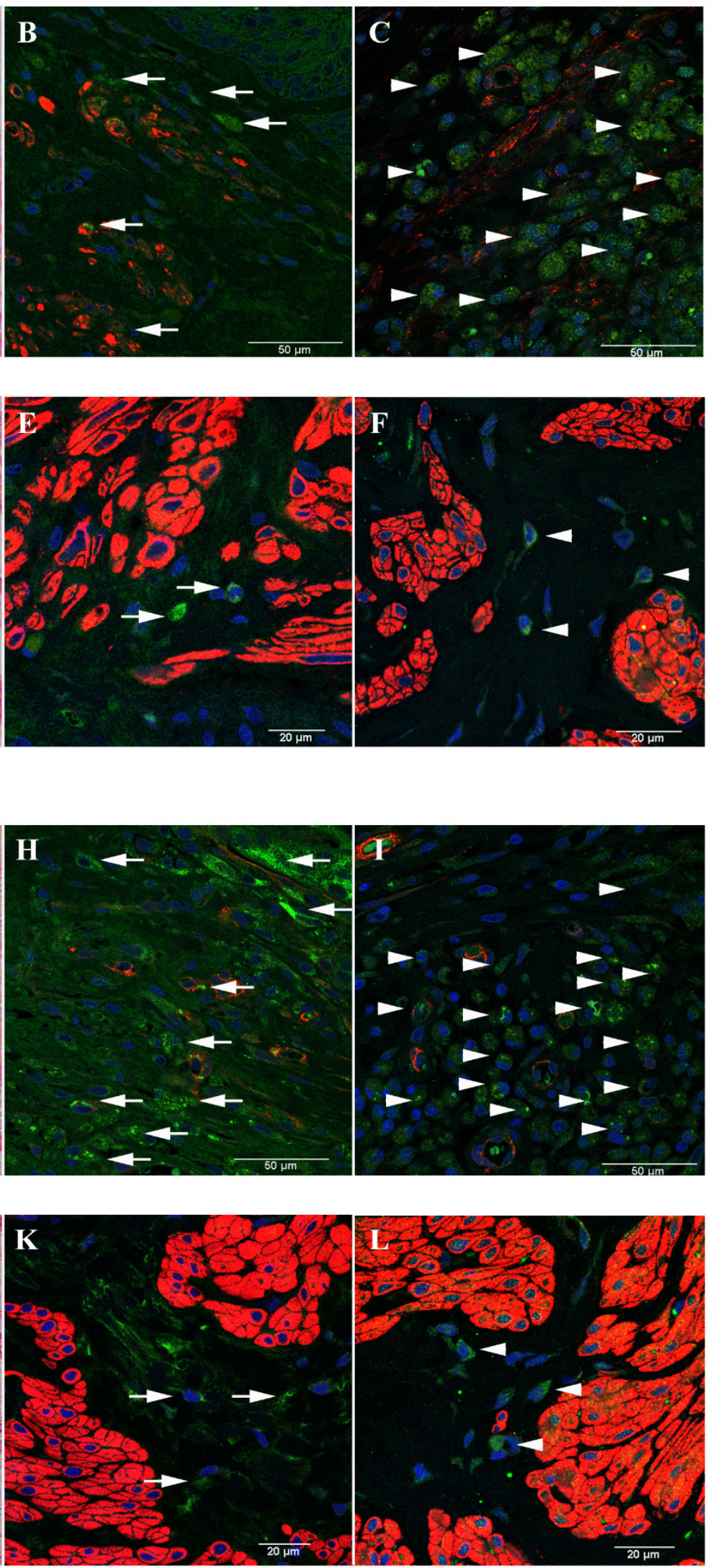

FIG. 6 
A Bladder Pressure $\quad 5$ min (I) Micturition Volume $2 \mathrm{ml}$ $2 \mathrm{ml}$

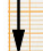

Structure Transplantation rats

B Bladder Pressure

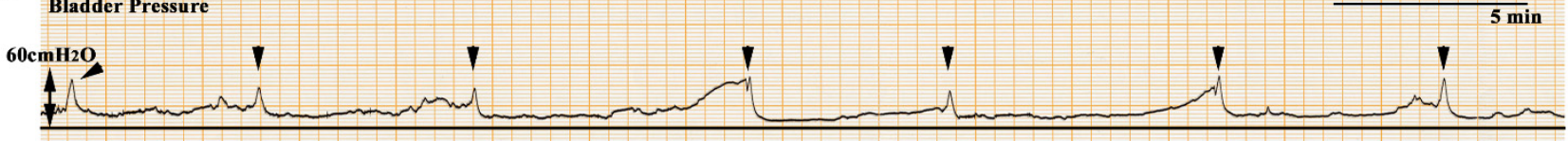

Micturition Volume

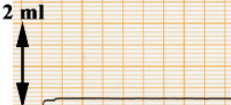

FIG. 7 
$\mathbf{A}$

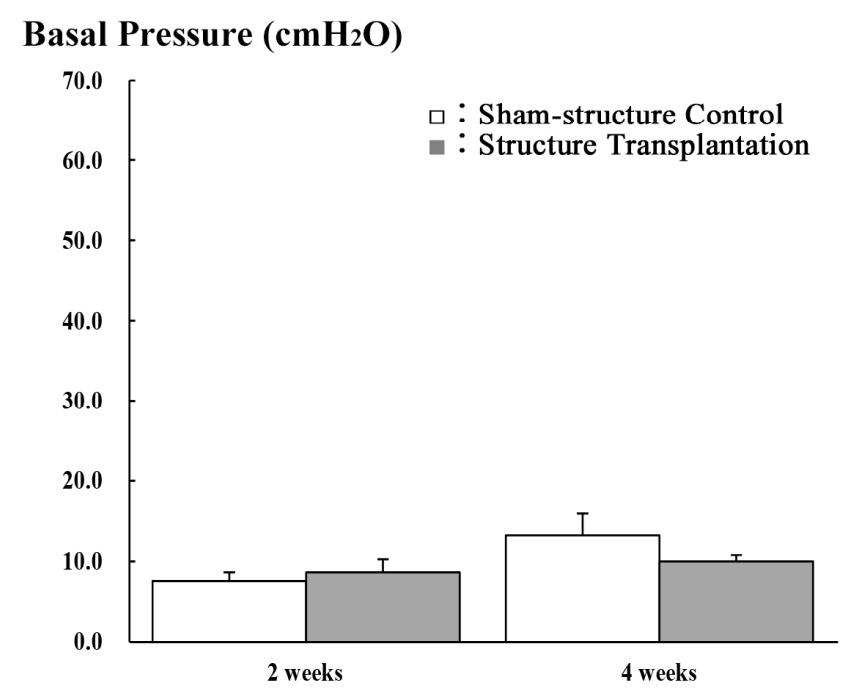

C

\section{Micturition Pressure $\left(\mathrm{cmH}_{2} \mathrm{O}\right)$}



E

\section{Micturition Volume (ml)}

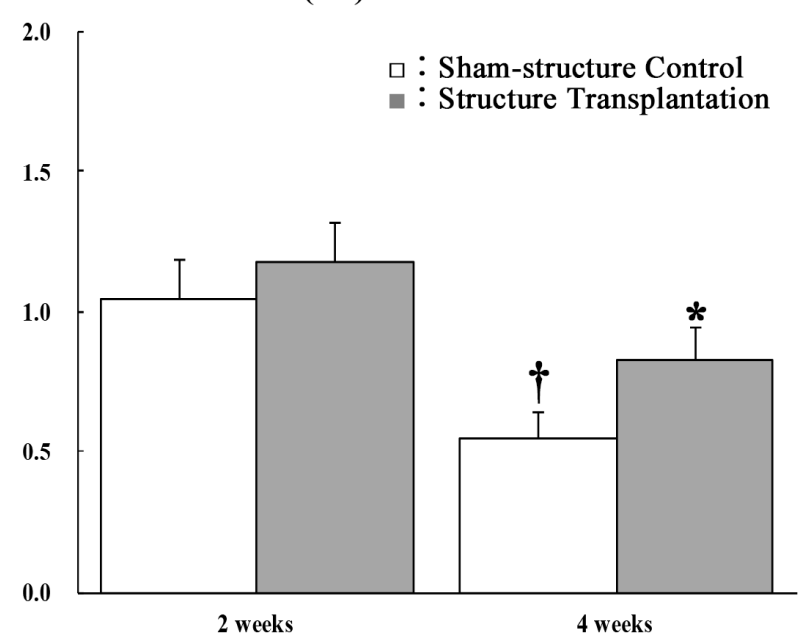

B

Threshold Pressure $\left(\mathrm{cmH}_{2} \mathrm{O}\right)$

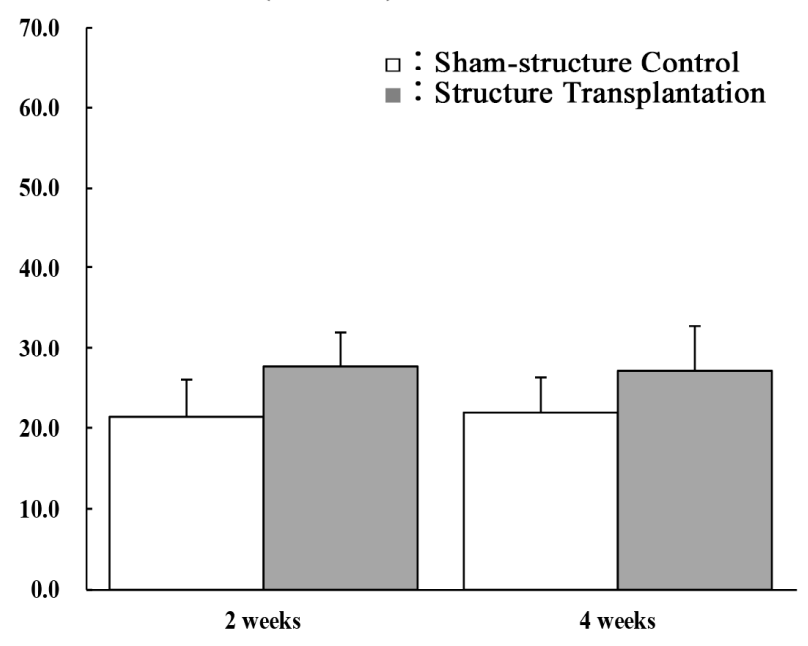

D

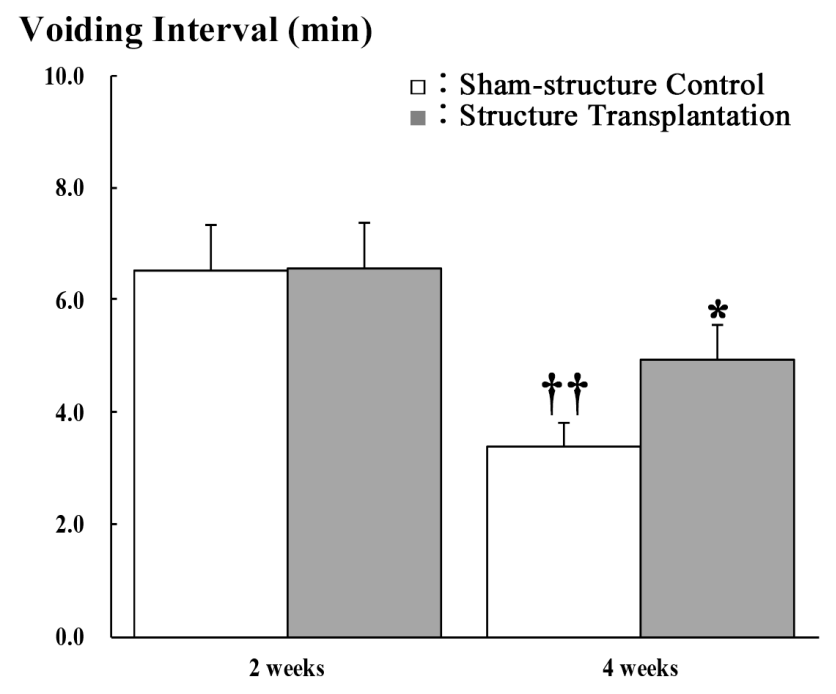

F

Residual Volume (ml)

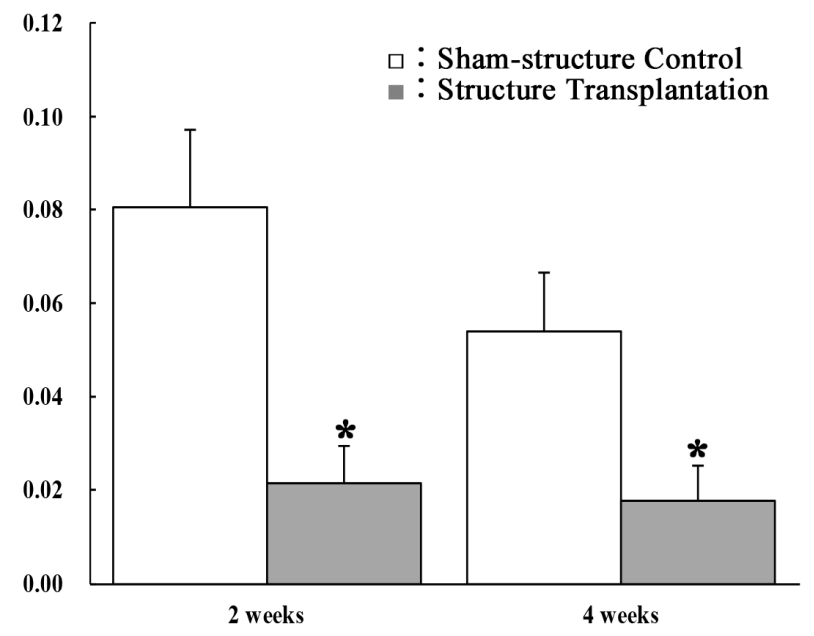

FIG. 8 